\title{
About a uni-cervical unicorn uterus case with vaginal septum
}

\section{Mapatano Shalamba E. ${ }^{1 *}$, Kalala Kanyinda L. ${ }^{1}$, Kiminyi Kalunga M. ${ }^{1}$, Nyakio Ngeleza O. ${ }^{1}$, Kanku Tudiakwile L. ${ }^{2}$, Mukwege Mukengere D. ${ }^{1}$}

\author{
${ }^{1}$ Department of Gynecology and Obstetrics, School of Medicine, Panzi Hospital, Evangelic University in Africa, \\ Bukavu, DRC \\ ${ }^{2}$ Department of Pediatrics, School of Medicine, Panzi Hospital, Evangelic University in Africa, Bukavu, DRC
}

Received: 16 October 2019

Revised: 22 November 2019

Accepted: 02 December 2019

\section{*Correspondence:}

Dr. Mapatano Shalamba E.,

E-mail: shalamba2mapatano@gmail.com

Copyright: (C) the author(s), publisher and licensee Medip Academy. This is an open-access article distributed under the terms of the Creative Commons Attribution Non-Commercial License, which permits unrestricted non-commercial use, distribution, and reproduction in any medium, provided the original work is properly cited.

\begin{abstract}
The unicorn uterus is a common uterine malformation after septate uteri, accounting for 30 to $50 \%$ of cases. We report a case of unicorn uterus with an associated vaginal septum discovered during the obstetrical examination of a consultant surgeon for hemorrhage, and exploration of the uterus revealed a uterus during a cesarean section. Maternal-fetal rescue for placenta previa. The interest of this case is to show the double embryological mechanism involved, unilateral aplasia of the muller ducts and the lack of resorption explaining this vaginal septum.
\end{abstract}

Keywords: Unicorn uterus, Utero-vaginal malformations, Vaginal septum, 3D ultrasound

\section{INTRODUCTION}

Uterine malformation (UM) is a congenital malformation abnormality resulting from a failure or arrest of the development of the female reproductive tract during embryogenesis. The incidence of UM is difficult to assess in the literature; it is estimated between 1 and $10 \%$ in the general population. ${ }^{1,2}$ In infertile women, the UM frequency is between 3 and $3.5 \% .^{3}$ The exact prevalence since many of these malformations are asymptomatic and imaging techniques such as 3D ultrasound, 3D hysterosonography and MRI have only been available for a few years. ${ }^{4-6}$ Uterine malformations seem to be diagnosed more frequently in certain groups of patients, for example during follow-up for infertility or for recurrent miscarriage. ${ }^{4}$ Among them, authors find uterine malformations, especially septate uteri that can be discovered during an imaging test or during a surgical procedure, or, as in our case, suspected during an obstetric examination and confirmed to uterine exploration during a cesarean section. The septate uterus is the most common uterine malformation, accounting for 30 to $50 \%$ of cases, followed by uterine malformations of the uterus bicorne and unicorn uterus. ${ }^{7}$ The vaginal longitudinal septa are isolated in $3-12 \%$ of cases, they are associated in $52 \%$ of cases to a septate uterus performing complete utero-vaginal septa and in $21 \%$ of cases to two half-uteri. ${ }^{8}$

Authors report here a case of vaginal septum with one blind hemi-vagina and the other part of the vagina giving access to the cervix discovered during the speculum obstetrical examination and of a unicorn uterus discovered during cesarean section indicated for placenta previa hemorrhagic at a great multipare in work.

\section{Organogenesis}

From the $7^{\text {th }}$ week of development, the female genital tract is differentiated: in the absence of anti-mullerian 
hormone, Wolff's channels regress and the Müller canals develop. This development has three phases: the migration of Müller's canals to the urogenital sinus $\left(6^{\text {th }}\right.$ to $9^{\text {th }}$ week); the opening of the lower third of the Müller canals forming the uterine cavity and the upper two thirds of the vagina ( $9^{\text {th }}$ to $13^{\text {th }}$ week); the resorption of the inter-Mullerian partition ( $13^{\text {th }}$ to $17^{\text {th }}$ week).

Most of the uterine malformations can be explained by a defect or a developmental failure during these three phases: the absence of migration or the incomplete caudal migration of the Müller canals to the urogenital sinus will be responsible for atrophy and/or complete uterine aplasia or not; a defect of fusion of the Müller's ducts leads to a uterine duplication (uterus didelphe, uterus bicorne); a defect in the resorption of the intermüllerian septum leads to a septate uterus.

A relatively constant element is the association of abnormalities of the genital apparatus and the urinary system because the embryogenesis of these two systems is intimately linked. ${ }^{1}$

\section{CASE REPORT}

This is Ms. X, a 35-year-old pregnant woman. In her obstetrical antecedents, she is gravida 9, pare 8, living children 3 , abortus 1 , death 4 ; whose chronology is as follows:

- $\quad 1^{\text {st }}$ pregnancy (in 2004): abortion;

- $\quad 2^{\text {nd }}$ pregnancy (in 2005): premature delivery by caesarean section with death 1 year later;

- $\quad 3^{\text {rd }}$ pregnancy (in 2007): premature vaginal delivery at home with death a few weeks later;

- $4^{\text {th }}$ pregnancy (in 2008): premature delivery by caesarean section whose newborn weighed $2000 \mathrm{~g}$ and died in the following weeks;

- $5^{\text {th }}$ pregnancy (in 2010): premature vaginal delivery of a stillbirth;

- $6^{\text {th }}$ pregnancy (in 2011): Pregnancy at term, vaginal delivery (fetal weight $3000 \mathrm{~g}$ );

- $\quad 7^{\text {th }}$ pregnancy (in 2012): Pregnancy at term, vaginal delivery (fetal weight $3100 \mathrm{~g}$ );

- $\quad 8^{\text {th }}$ pregnancy (in 2016): Pregnancy at term, delivery by caesarean section (fetal weight $3500 \mathrm{~g}$ );

- $\quad 9^{\text {th }}$ pregnancy (in 2019): current pregnancy.

She was transferred from a local hospital center for better management of bleeding on non-term pregnancy with unknown last menstrual period (L.M.P).

The beginning of the symptomatology was about 3 days before our consultation by vaginal bleeding made of bright red blood, painless, capricious, average abundance without triggering factors nor sedatives for which she consulted this Hospital Center, but the persistence of the associated bleeding to the lombo-pelvic pain that motivated the transfer for better care.

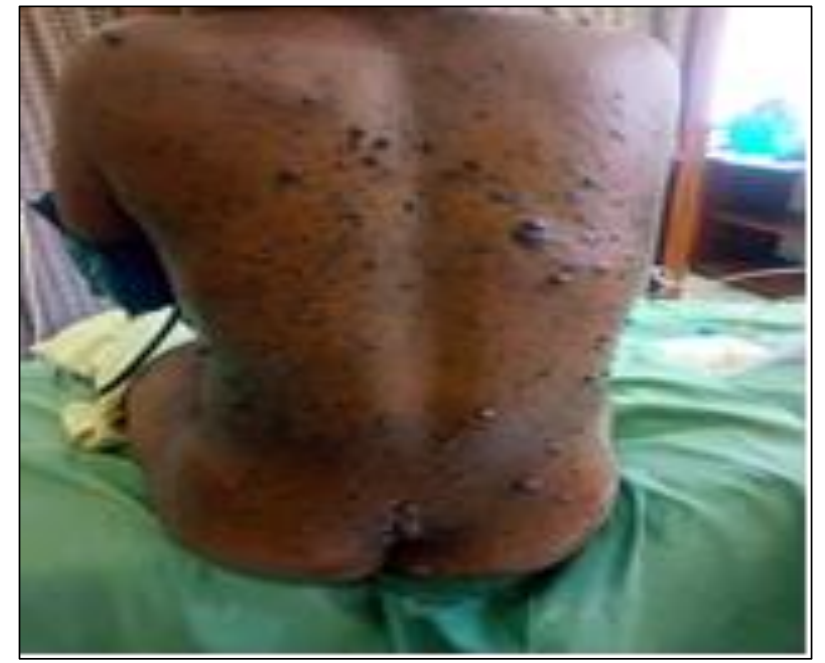

Figure 1: Cutaneous lesion.

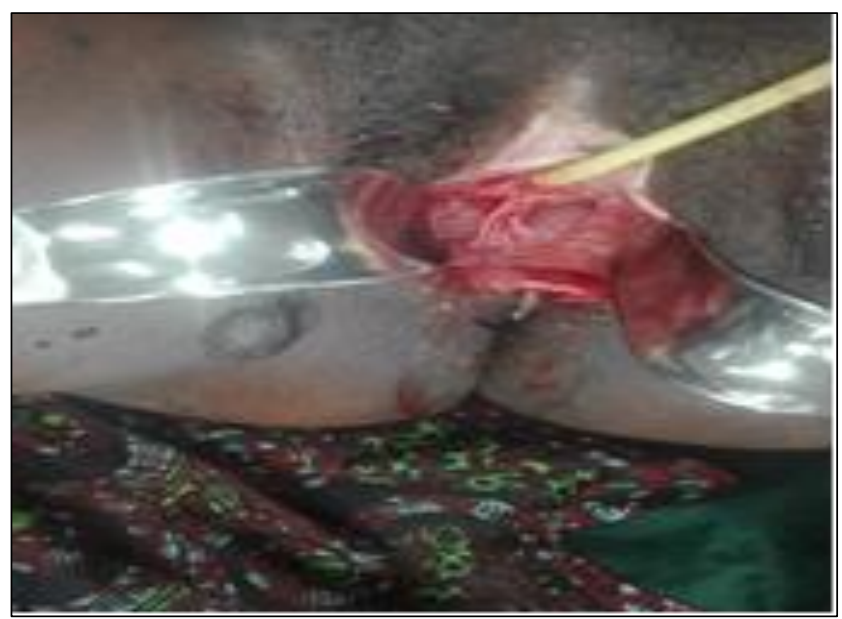

Figure 2: Vaginal septum.

Our physical examination noted a blood pressure of 90/60 $\mathrm{mmHg}$ with a pulse rate of 110 and a respiratory rate of 30 cycles/min, a general condition impaired by intense asthenia with poor mucocutaneous cutaneous staining and a rash all over the body that dates back to several years (Figure 1). Her obstetrical examination revealed a uterine height of $29 \mathrm{~cm}$, a poorly appreciated presentation, position I and 128 beats on auscultation of fetal heart sounds.

The vulva was stained with bright red blood and examination at the valves had revealed a vagina with vertical septum or active bleeding, abundant ex utero was noted to the right of the septum in the hemi-vagina which gives access to the cervix and a blind vaginal cavity to the left of the septum (Figure 2). The vaginal touch was not done.

Authors concluded a haemorrhage of the 3rd trimester complicated by a state of shock with as suspected etiology a low inserted placenta hemorrhagic on a ground of a vaginal malformation. 
Ultrasonography revealed an active fetal mono fetal pregnancy of 30 weeks and 4 days, weight estimate at $1650 \mathrm{~g}$ in cephalic presentation with posterior placenta inserted posterior type III Bessis.

The rapid blood count, blood group, and rhesus blood count revealed a blood group $\mathrm{O}$ Rhesus + and a hemoglobin at $94 \mathrm{~g} / \mathrm{L}$ with HIV testing after counseling that was negative.

Despite the resuscitation measures taken and the blood transfusion given, it was noted that there was still abundant bleeding of bright red blood, and an emergency caesarean section was decided for maternal-fetal maternal rescue.

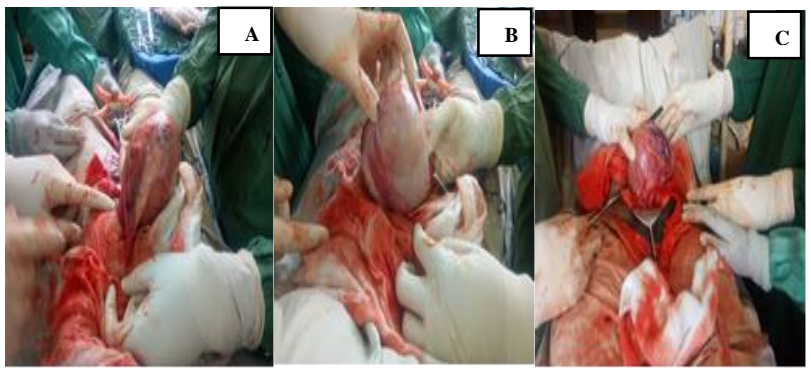

Figure 3: Different facets of the uterus during caesarean section. (A) Horn of the uterus, (B) uterus with right appendix, (C) Uterus showing the absence of left appendix.

This intervention allowed us to extract cephalic a newborn whose score was rated at 4 to $1 \mathrm{~min}, 5$ to $5 \mathrm{~min}$ and 6 to $10 \mathrm{~min}$, weighing $1700 \mathrm{~g}$, sex:M, with as an observation intraoperatively a posterior low placenta inserted at the origin of the bleeding and the exploration of the uterus had allowed us to note a uterus possessing only a right ovary and nothing on the left (Figure 3 ).

No therapeutic gesture aimed at the malformation has been performed. The postpartum was unparalleled and an ultrasound of the urinary shaft was requested for screening for any associated urinary malformations returned without abnormalities. The operative sequences were simple and the new-born mutated in neonatology had a simple evolution.

\section{DISCUSSION}

The prevalence of congenital uterine abnormalies in the population is estimated to be between 1 and $4 \%$ according to the studies. ${ }^{2,7}$ Although this prevalence remains inaccurate given the asymptomatic characteristics of these malformations. The diagnosis is most frequently made in patients treated for infertility, recurrent miscarriage or premature labor, in which case the patient was asymptomatic in her life and during this pregnancy and the diagnosis was made at the clinical examination at admission for the management of her delivery. However, it remains important to discuss the diagnosis of adolescents who consult for primary amenorrhea, dysmenorrhea or dyspareunia.

Organogenesis of the genitourinary tract can be used to interpret and classify genital malformations. Four phases can be schematized: the first urinary phase $\left(3^{\text {rd }}, 4^{\text {th }}\right.$ and $5^{\text {th }}$ weeks) involves the formation of Wolff's ducts and their progression towards the cloaca, the development of ureteral buds towards renal blastemas; the second phase, genital and urinary $\left(6^{\text {th }}, 7^{\text {th }}, 8^{\text {th }}\right.$ and $9^{\text {th }}$ weeks $)$ involves the completion of the urinary tract by the ascension and rotation of the kidneys, from the $9^{\text {th }}$ week the urinary organogenesis is thus completed; the formation of Müller's canals and their progression to the genital sinus may begin; the third phase, genital, of the joining of the two channels of Müller spreads on the $10^{\text {th }}, 11^{\text {th }}$ and $12^{\text {th }}$ weeks; this phase is responsible for the external morphology of the genital tract; the fourth and last phase is that of the resorption of the Müller canal partition wall ( $13^{\text {th }}$ to $17^{\text {th }}$ week). The resorption begins at the isthmus before the end of the crouching phase, rapidly extends downwards and slowly upwards. This resorption phase is responsible for the internal morphology of the genital tract. The type of malformations is related to the date of onset of the endogenous or exogenous teratogenic agent during organogenesis:

Between three and six weeks, the Müller Canal does not exist yet. Uterine aplasia, unicornous uterus with unilateral renal agenesis; at the sixth week, the Müller canal develops.

An abnormality that appears between six and nine weeks of age will result in a pseudo unicorn uterus. Between ten and thirteen weeks, Müller's two canals are close to the center line. The anomalies observed are a defect of fusion of the two Müller's ducts, at the origin of the bicorne uteri; after thirteen weeks, there is a disturbance of the resorption of the septum at the origin of the septate uteri.

The classification of uterine malformations, the most used in France is the classification of Muset, established in $1964 .{ }^{8}$ The international classification is that of the American Fertility Society (AFS) of 1988. It is the most used in the literature currently.

Classification of the American Fertility Society (AFS) 1988 includes seven groups of malformations

- Class1: Bilateral uterine aplasia

- Class 2: Complete unilateral uterine aplasia or true unicorn uterus

- Class 3: the bi-cervical bicorne uteri

- Class 4: cervical unicorne uteri

- Class 5: absence of resorption of the inter-Mullerian septum: septate uteri

- Class 6: absence of resorption of inter-Mullerian septum: Uterus arched or spur 
- Class 7: uterine abnormality due to diethylstilbestrol outside pregnancy.

The utero-vaginal malformation can be discovered: as part of a primary amenorrhea assessment; disabling primary dysmenorrhea; infertility; repeated premature births or dyspareunia.

The abnormality discovered during the clinical examination may be: absence of vagina; a bulge of the lateral wall of the vagina; a vaginal septum; cervical bifurcation or even absence of cervix. ${ }^{9}$ During pregnancy, it may be necessary to diagnose a malformation: during a premature termination of pregnancy; in front of a repeated dystocic presentation; during a delivery accident (hemorrhage or retention); and more rarely before the occurrence of accidents: hemoperitoneum by rupture of a rudimentary uterine horn gravid of a pseudo unicorn uterus; or acute abdominal syndrome, by torsion of a gravid uterus (absence of congenital ligament).In this case, the diagnosis was made during the management of the bleeding of the third trimester of pregnancy that is the placenta praevia hemorrhagic. In the assessment of a genital malformation. The different techniques used are 2D or 3D ultrasound, hysterosonography, hysterosalpingography, MRI, hysteroscopy and laparoscopy. ${ }^{10}$ These different techniques can be combined with each other. 3D ultrasound and MRI are currently the techniques showing the best results in terms of sensitivity and specificity. ${ }^{11,12}$ The assessment of uterine malformations should be supplemented by renal imaging to detect the frequently associated malformations of the urinary tract.

Ultrasound should always be performed because it is the only exploration that allows accurate endo- and exouterine evaluation. In routine practice, his sensitivity remains small (30 to $40 \%$ ) because sonographer dependent.

Laparoscopy should be performed exceptionally and reserved only for cases where the diagnosis remains hesitant between septate uterus and bicornous uterus. In this case, it is a unicorn uterus that results from the unilateral aplasia of muller canals or (AFS type II)..$^{9,13}$

The true unicorn uterus (complete unilateral aplasia)

\section{Authors find in 2D ultrasound and 3D ultrasound}

- A strongly latero-deviated uterus: the axis of the vaginal probe is very oblique with respect to the median line

- A reduced uterine volume in length and thickness, which often has an ellipsoid appearance

- In 3D ultrasound, the reconstructed frontal section finds a canalicular cavity in flames, with loss of the triangular aspect. ${ }^{14}$
In this case, the diagnosis was made intraoperatively during the exploration of the uterus after an obstetrical examination which made it possible to visualize a vaginal septum with a pregnant consultant for genital hemorrhage.

The upper two-thirds of the vagina having the same embryological origin as the uterus, uterine malformations are often associated with high vaginal malformations. ${ }^{15}$

Embryologically, the vaginal septum is related to a lack of resorption of the lower part of Muller's canals, isolated vaginal septa are rare, they are often associated for the majority of them with uterine septa producing a uterine septum. Vaginal complete within the framework of the septate uteri with only one cervical mass or that of the uterine hemi-matrices with two distinct cervical massifs. ${ }^{16,17}$

In this case, two opposite mechanisms are involved, on the one hand authors have a unilateral aplasia of the Muller ducts giving the unicorn uterus and on the other authors have a resorption defect of the lower part of these same channels giving a vaginal septum with two hemivaginas including one right which is one-eyed.

\section{CONCLUSION}

Congenital uterine malformations are relatively frequent and often asymptomatic. Their exact incidence remains difficult to assess. They can manifest as gynecological disorders or have an impact on reproduction. Each clinician should look for utero-vaginal malformation in the presence of primary amenorrhoea, abdominal pain, recurrent miscarriage, and some adverse obstetric outcomes. Their diagnosis and assessment of a prognosis require a specific assessment (3D ultrasound, hysterosonography, MRI).

Although many cases are crowned with pregnancy, psychological management may be necessary for some patients, particularly for malformations that are incompatible with a pregnancy such as MRKH and/or have an impact on sexuality. It should be remembered that when diagnosing uterine malformation, urinary tract imaging should be performed because of frequent associated abnormalities.

\section{ACKNOWLEDGMENTS}

Authors would like to thanks the medical director of Panzi Hospital who allowed the publication of this case. Also, thanks to patient to whom we did this case study.

Funding: No funding sources Conflict of interest: None declared

Ethical approval: The clearance committee of Evangelic University in Africa approved this study 


\section{REFERENCES}

1. Itchimouh $\mathrm{S}$, Khabtou $\mathrm{K}$, Mahdaoui $\mathrm{S}$, Boufettal $\mathrm{H}$, Samouh N. Uterine rupture in a patient with bicornuate uterus at 12 weeks of amenorrhea: about a case. The Pan African Med J. 2016;24:153.

2. Nahum GG. Uterine anomalies. How common are they, and what is their distribution among subtypes? J Reprod Med. 1998;43:877-87.

3. Saravelos SH, Cocksedge KA, Li TC. Prevalence and diagnosis of congenital uterine anomalies in women with reproductive failure: a critical appraisal. Human Reprod Update. 2008;14:415-29.

4. Raga F, Bauset C, Remohi J, Bonilla-Musoles F, Simón C, Pellicer A. Reproductive impact of congenital Müllerian anomalies. Human Reproduction (Oxford, England). 1997;12(10):2277-81.

5. Troiano RN, McCarthy SM. Mullerian duct anomalies: imaging and clinical issues. Radiol. 2004;233(1):19-34.

6. Poncelet C, Aissaoui F. Malformations utérines et reproduction. Gynécol Obstét Fertil. 2007;35(9):821-5.

7. Acien P. Incidence of müllerian defects in fertile and infertile women. Human Repro (Oxford, England). 1997;12(7):1372-6.

8. Golan A, Langer R, Neuman M, Wexler S, Segev E, David MP. Obstetric outcome in women with congenital uterine malformations. The J Repro Med. 1992;37(3):233-6.

9. Society TAF. The American Fertility Society classifications of adnexal adhesions, distal tubal occlusion, tubal occlusion secondary to tubal ligation, tubal pregnancies, Müllerian anomalies and intrauterine adhesions. Fertil Steril. 1988;49(6):944-55.

10. Deutch TD, Abuhamad AZ. The role of 3-dimensional ultrasonography and magnetic resonance imaging in the diagnosis of Müllerian duct anomalies: a review of the literature. J Ultrasound Med. 2008;27(3):413-23.

11. Salim R, Woelfer B, Backos M, Regan L, Jurkovic D. Reproducibility of three-dimensional ultrasound diagnosis of congenital uterine anomalies. Ultrasound Obstet Gynecol. 2003;21(6):578-82.

12. Woelfer B, Salim R, Banerjee S, Elson J, Regan L, Jurkovic D. Reproductive outcomes in women with congenital uterine anomalies detected by threedimensional ultrasound screening. Obstet Gynecol. 2001;98(6):1099-103.

13. Airoldi J, Berghella V, Sehdev H, Ludmir J. Transvaginal ultrasonography of the cervix to predict preterm birth in women with uterine anomalies. Obstet Gynecol. 2005;106(3):553-6.

14. Acién P. Reproductive performance of women with uterine malformations. Human Repro. 1993;8(1):122-6.

15. Golan A, Langer R, Neuman M, Wexler S, Segev E, David MP. Obstetric outcome in women with congenital uterine malformations. The J Repro Med. 1992;37(3):233-6.

16. Golan A, Langer R, Wexler S, Segev E, Niv D, David MP. Cervical cerclage-its role in the pregnant anomalous uterus. Int J Fert. 1990;35(3):164-70.

17. Levaillant JM. Diagnostiquer une malformation utérine. J de Gynécol Obstét Biol Repro. 2007;36:6-12.

Cite this article as: Shalamba ME, Kanyinda KL, Kalunga KM, Ngeleza NO, Tudiakwile KL, Mukengere MD. About a uni-cervical unicorn uterus case with vaginal septum. Int J Reprod Contracept Obstet Gynecol 2020;9:404-8. 